\title{
Use of Comet Assay as an Efficient Biomarker for Plant Biomonitoring and Phytomanagement of Contaminated Sites
}

\author{
Pourrut Bertrand $^{1}$, Boccia Priscilla ${ }^{2}$, Sturchio Elena ${ }^{2}$ \\ ${ }^{1}$ Yncrea Hauts de France - ISA Lille - LGCgE \\ 48 boulevard Vauban, Lille, France \\ bertrand.pourrut@yncrea.fr \\ ${ }^{2}$ Italian Workers' Compensation Authority (INAIL), Department of Technological Innovation and Safety of Plants \\ Product and Anthropic Settlements (DIT) \\ Via R. Ferruzzi 38, Roma, Italy \\ p.boccia@inail.it; e.sturchio@inail.it
}

\section{Extended Abstract}

During the last decade plants have been increasingly used in ecotoxicological studies and environmental biomonitoring. In order to evaluate the impact of stress (biotic or abiotic) on plants, it is important to evaluate their health. This can be realized at the macroscopic scale (growth, dry or fresh weight...) or at the molecular scale, using biomarkers. During the same period, the application of the comet assay has been established as one of the most interesting techniques in eco-genotoxicology. It is a rapid, versatile, sensitive and relatively inexpensive method for measuring DNA damages and repairs in individual cells. The aim of this work was to evaluate the interest of the comet assay to monitor pollutant impacts on higher plants growing on contaminated sites and to select plant species to remediate contaminated areas.

In a first study, we investigated the potential impacts of contaminants from a hazardous waste site, in a controlled environment, on Vicia faba, as a bioindicator plant. Soil samples were collected from a former industrial area in Italy and their phytotoxicity and genotoxicity were investigated. In the case of the controlled environment we evaluated the environmental damage after a simulated accidental release of toxic substances in soil. In this case we evaluated the contamination effects on soil-plant system and detected DNA damages by short-term genotoxicity tests (comet assay and micronuclei tests) performed on polluted soils and on gravitational water. Our studies demonstrated that the comet assay is a sensitive, rapid and cost-effective technique for the detection of DNA damage, which is ideally suited as a biomarker of genotoxicity for biomonitoring.

In a second study, we investigated sub-lethal effects of contaminated soil exposure on three plant species which have been described as good candidates for phytomanagement of contaminated areas: ryegrass (Lolium perenne), clover (Trifolium repens) and miscanthus (Miscanthus $x$ giganteus). Plants were grown on soils contaminated with heavy metals by a former lead smelter in Northern France. We analyzed several biomarkers (oxidative stress, lipid peroxidation, photosynthetic pigments) to evaluate plant health and we compared them with DNA degradation measured by comet assay. Our results clearly demonstrated different metal tolerances among these plants. Despite its potential to stabilize pollutants in soils, ryegrass plants exhibited high level of oxidative stress, lipid degradation and DNA stand breaks. These results challenge the suitability of this plant for a long-term management of contaminated soils. In the other hands, miscanthus plants showed little effects of metals, event at extremely high concentrations. This confirm this plant as good candidate for phytomanagement. For each plants, strong correlations were founded between comet assay results and other biomarkers after short term exposure and between comet assay and macroscopic markers (growth and weight) after long term exposure. These results confirm the interest of the use of comet assay to select potential candidate plants for phytoremediation.

\section{References}

[1] E. Sturchio, P. Boccia, C. Meconi, M. Zanellato, S. Marconi, C. Beni, R. Aromolo, A. Ciampa, and M. Valentini, "Methods to investigate arsenic contamination on soil-plant system," Chem. Ecol., vol. 27, pp. 1-12, 2011. 